\title{
NUMERICAL EVALUATION OF SPECIAL POWER SERIES INCLUDING THE NUMBERS OF LYNDON WORDS: AN APPROACH TO INTERPOLATION FUNCTIONS FOR APOSTOL-TYPE NUMBERS AND POLYNOMIALS*
}

\author{
IREM KUCUKOGLU ${ }^{\dagger}$ AND YILMAZ SIMSEK ${ }^{\ddagger}$
}

\author{
Dedicated to Walter Gautschi on the occasion of his 90th birthday
}

\begin{abstract}
Because the Lyndon words and their numbers have practical applications in many different disciplines such as mathematics, probability, statistics, computer programming, algorithms, etc., it is known that not only mathematicians but also statisticians, computer programmers, and other scientists have studied them using different methods. Contrary to other studies, in this paper we use methods associated with zeta-type functions, which interpolate the family of Apostol-type numbers and polynomials of order $k$. Therefore, the main purpose of this paper is not only to give a special power series including the numbers of Lyndon words and binomial coefficients but also to construct new computational algorithms in order to simulate these series by numerical evaluations and plots. By using these algorithms, we provide novel computational methods to the area of combinatorics on words including Lyndon words. We also define new functions related to these power series, Lyndon words counting numbers, and the Apostoltype numbers and polynomials. Furthermore, we present some illustrations and observations on approximations of functions by rational functions associated with Apostol-type numbers that can provide ideas on the reduction of the algorithmic complexity of these algorithms.
\end{abstract}

Key words. Lyndon words, special numbers and polynomials, Apostol-type numbers and polynomials, arithmetical function, interpolation function, zeta type function

AMS subject classifications. 03D40, 05A15, 11A25, 11B68, 11B83, 11S40, 11Y16, 65Q30, 68R15

1. Introduction. The main motivation of this article is to construct not only new formulas for special power series representations involving the Lyndon words counting numbers and the Apostol-type numbers and polynomials but also algorithms for the numerical computations using these formulas. In addition, we define new functions including these infinite series and Lyndon words counting numbers, and their numerical values are computed and plotted with the help of constructed computational algorithms.

Let us start by giving a brief description of the Lyndon words, one of the main motivations of this article, and the related definitions of the generating functions of the numbers that count them. The $k$-ary Lyndon words of length $n$, represented by a primitive necklace consisting of $n$ beads of $k$ different colors, are the lexicographically smallest elements in the set derived from all primitive words having length $n$ over the $k$-letter alphabet. Here, primitive words means that a word cannot be written as a positive power of its subwords. For instance, let us consider the alphabet $\{0,1\}$. All 2-ary Lyndon words of length 5 which are derived from this alphabet are given as follows: $\{00001,00011,00101,00111,01011,01111\}$. It is clear that the elements of this finite set are primitive words (cf. $[3,4,6,7,11]$ and the references cited therein).

${ }^{*}$ Received February 1, 2018. Accepted September 13, 2018. Published online on January 8, 2019. Recommended by G. V. Milovanović. The present paper was supported by Scientific Research Project Administration of Akdeniz University (with Project Number: FBA-2018-3292).

${ }^{\dagger}$ Department of Engineering Fundamental Sciences, Faculty of Engineering, Alanya Alaaddin Keykubat University TR-07425 Antalya, Turkey (irem. kucukogluealanya.edu.tr).

${ }^{\ddagger}$ Department of Mathematics, Faculty of Science University of Akdeniz TR-07058 Antalya, Turkey (ysimsek@akdeniz.edu.tr). 


\section{ETNA}

Kent State University and

Johann Radon Institute (RICAM)

In this paper, $L_{k}(n)$ denotes the numbers of $k$-ary Lyndon words of length $n$. These numbers are computed by the following formula (cf. $[3,4,6,7])$ :

$$
L_{k}(n)=\frac{1}{n} \sum_{d \mid n} \mu\left(\frac{n}{d}\right) k^{d}
$$

where the sum is over all positive divisors of $n$ and $\mu$ is the Möbius function, which is an arithmetic function defined by (cf. [2])

$$
\mu(1)=1
$$

and for $n>1$ if we write $n=p_{1}^{a_{1}} p_{2}^{a_{2}} \ldots p_{k}^{a_{k}}$, where $p_{1}, p_{2}, \ldots, p_{k}$ are $k$ distinct primes, then

$$
\mu(n)=\left\{\begin{array}{cl}
(-1)^{k} & \text { if } a_{1}=a_{2}=\cdots=a_{k}=1 \\
0, & \text { if } n \text { is the product of non-distinct primes (i.e., } n \text { is not square-free). }
\end{array}\right.
$$

The paper is organized as follows. In Section 2 we give some auxiliary results. Two new special power series, including the numbers of Lyndon words and binomial coefficients, are defined in Section 3. We give new relations and identities related to these series including zeta-type functions, the Apostol-type numbers of order $k$, and the Apostol-type polynomials of order $k$. Section 4 is devoted to some algorithms for computing the values of the functions representing these special power series by using a modification of recurrence formulas for the family of Apostol-type numbers and polynomials of order $k$. Finally, in Section 5, by using our algorithms, we provide some numerical evaluations and plots. Furthermore, we present some illustrations and observations for the approximation by rational functions of functions representing special power series that can provide ideas on the reduction of the algorithmic complexity of our algorithms.

2. Some auxiliary results. We start this section with descriptions and formulas for the Apostol-type numbers and polynomials, including their generating functions and their interpolation functions.

The Apostol-type numbers and polynomials of order $k$ have been defined by the second author [14] as

$$
F_{w}(t ; \lambda ; k)=\frac{1}{\left(\lambda e^{t}+\lambda^{-1} e^{-t}+2\right)^{k}}=\sum_{n=0}^{\infty} W_{n}^{(k)}(\lambda) \frac{t^{n}}{n !}
$$

and

$$
G_{w}(t, x, k ; \lambda)=e^{t x} F_{w}(t ; \lambda ; k)=\sum_{n=0}^{\infty} W_{n}^{(k)}(x ; \lambda) \frac{t^{n}}{n !},
$$

respectively, where $k$ is a non-negative integer and $\lambda$ is a real or complex parameter. A relation between the numbers $W_{n}^{(k)}(\lambda)$ and the polynomials $W_{n}^{(k)}(x ; \lambda)$ is given in the following theorem [14]: 
THEOREM 2.1. We have

$$
W_{n}^{(k)}(x ; \lambda)=\sum_{m=0}^{n}\left(\begin{array}{c}
n \\
m
\end{array}\right) x^{n-m} W_{m}^{(k)}(\lambda) .
$$

Setting $x=0$ in (2.1), we obtain

$$
W_{n}^{(k)}(\lambda)=W_{n}^{(k)}(0 ; \lambda)
$$

A computational formula for the numbers $W_{n}^{(k)}(\lambda)$ is given by the next theorem [15]:

THEOREM 2.2.

$$
W_{n}^{(c+d)}(\lambda)=\sum_{m=0}^{n}\left(\begin{array}{c}
n \\
m
\end{array}\right) W_{m}^{(c)}(\lambda) W_{n-m}^{(d)}(\lambda)
$$

By using the above formula for the numbers $W_{n}^{(k)}(\lambda)$, some numerical values of these numbers are obtained (cf. [15]):

$$
\begin{aligned}
& W_{0}^{(2)}(\lambda)=\frac{\lambda^{2}}{(\lambda+1)^{4}}, \quad W_{1}^{(2)}(\lambda)=\frac{2 \lambda^{2}(1-\lambda)}{(\lambda+1)^{5}}, \quad W_{2}^{(2)}(\lambda)=\frac{4 \lambda^{2}\left(\lambda^{2}-3 \lambda+1\right)}{(\lambda+1)^{6}} \\
& W_{0}^{(3)}(\lambda)=\frac{\lambda^{3}}{(\lambda+1)^{6}}, \quad W_{1}^{(3)}(\lambda)=\frac{3 \lambda^{3}(1-\lambda)}{(\lambda+1)^{7}}, \quad W_{2}^{(3)}(\lambda)=\frac{3 \lambda^{3}\left(3 \lambda^{2}-8 \lambda+3\right)}{(\lambda+1)^{8}} .
\end{aligned}
$$

It should be noted that

$$
W_{n}(\lambda)=W_{n}^{(1)}(\lambda) \quad \text { and } \quad W_{n}(x ; \lambda)=W_{n}^{(1)}(x ; \lambda) .
$$

The next theorem states a recurrence relation for the numbers $W_{n}(\lambda)$ (cf. [14]):

THEOREM 2.3. Let $n$ be a positive integer, and let

$$
W_{0}(\lambda)=\frac{\lambda}{(\lambda+1)^{2}}
$$

Then the recurrence relation

$$
2 W_{n}(\lambda)+\lambda \sum_{m=0}^{n}\left(\begin{array}{l}
n \\
m
\end{array}\right) W_{m}(\lambda)+\lambda^{-1} \sum_{m=0}^{n}(-1)^{n-m}\left(\begin{array}{l}
n \\
m
\end{array}\right) W_{m}(\lambda)=0
$$

holds.

By (2.3), the following numerical values of the numbers $W_{n}(\lambda)$ can be found:

$$
\begin{aligned}
& W_{1}(\lambda)=-\frac{\lambda(\lambda-1)}{(\lambda+1)^{3}}, \quad W_{2}(\lambda)=\frac{\lambda\left(\lambda^{2}-4 \lambda+1\right)}{(\lambda+1)^{4}}, \\
& W_{3}(\lambda)=-\frac{\lambda\left(\lambda^{3}-11 \lambda^{2}+11 \lambda-1\right)}{(\lambda+1)^{5}}, \ldots
\end{aligned}
$$

The well-known Apostol-Bernoulli polynomials $\mathcal{B}_{n}^{(k)}(x ; \lambda)$ and the Apostol-Euler polynomials $\mathcal{E}_{n}^{(k)}(x ; \lambda)$ can be expressed in terms of the polynomials $W_{n}^{(k)}(x ; \lambda)$. Indeed, the following relations hold (cf. [8]):

$$
\mathcal{B}_{n+2 k}^{(2 k)}(x ; \lambda)=\frac{(-1)^{k}(n+2 k)_{2 k}}{\lambda^{k}} W_{n}^{(k)}(x-k ;-\lambda),
$$




$$
\mathcal{E}_{n}^{(2 k)}(x ; \lambda)=\left(\frac{4}{\lambda}\right)^{k} W_{n}^{(k)}(x-k ; \lambda) .
$$

Otherwise, these polynomials of order $k, \mathcal{B}_{n}^{(k)}(x ; \lambda)$ and $\mathcal{E}_{n}^{(k)}(x ; \lambda)$, are defined by the generating functions

$$
\left(\frac{t}{\lambda e^{t}-1}\right)^{k} e^{x t}=\sum_{n=0}^{\infty} \mathcal{B}_{n}^{(k)}(x ; \lambda) \frac{t^{n}}{n !}
$$

and

$$
\left(\frac{2}{\lambda e^{t}+1}\right)^{k} e^{x t}=\sum_{n=0}^{\infty} \mathcal{E}_{n}^{(k)}(x ; \lambda) \frac{t^{n}}{n !}
$$

(cf. $[1,5,12,13,16,17,18])$.

REMARK 2.4. For other properties, relations, and identities related to the numbers $W_{n}(\lambda)$ and $W_{n}^{(k)}(\lambda)$ as well as to the polynomials $W_{n}(x ; \lambda)$ and $W_{n}^{(k)}(x ; \lambda)$, the reader can consult $[8,9,14,15]$.

In addition to the above preliminaries about the Apostol-type numbers and polynomials $W_{n}^{(k)}(\lambda)$ and $W_{n}^{(k)}(x ; \lambda)$, we now give their interpolation functions

$$
\zeta_{w}(s, k ; \lambda)=\sum_{m=0}^{\infty}(-1)^{m}\left(\begin{array}{c}
m+2 k-1 \\
m
\end{array}\right) \frac{\lambda^{m+k}}{(m+k)^{s}}
$$

and

$$
\zeta_{w}(s, x, k ; \lambda)=\sum_{m=0}^{\infty}(-1)^{m}\left(\begin{array}{c}
m+2 k-1 \\
m
\end{array}\right) \frac{\lambda^{m+k}}{(x+m+k)^{s}},
$$

respectively, where $\lambda, s \in \mathbb{C}$ with $|\lambda|<1$ and $\Re(s)>1$ (cf. [9]). Special cases of the above functions, related to the Hurwitz-Lerch zeta function, are given in [9]. For $n \in \mathbb{N}$, we also have (cf. [9])

$$
\begin{aligned}
\zeta_{w}(-n, k ; \lambda) & =W_{n}^{(k)}(\lambda), \\
\zeta_{w}(-n, x, k ; \lambda) & =W_{n}^{(k)}(x ; \lambda) .
\end{aligned}
$$

3. Two special power series including the numbers of Lyndon words. In this section, we define two new special power series involving the numbers of Lyndon words and binomial coefficients. We give relations between these series and zeta-type functions. In the next section, we give algorithms for computing the values of these series by using modifications of the corresponding recurrence formulas for the numbers $W_{n}^{(k)}(\lambda)$.

First, we define a power series involving the numbers $L_{k}(n)$ and the binomial coefficients.

Definition 3.1. Let $\lambda \in \mathbb{C}$ with $|\lambda|<1$, and let $n$ be a positive integer. We define

$$
\mathcal{G}(\lambda, n, k)=\sum_{m=0}^{\infty}\left(\begin{array}{c}
-2 k \\
m
\end{array}\right) L_{m+k}(n) \lambda^{m+k}
$$

Since

$$
\left(\begin{array}{c}
-2 k \\
m
\end{array}\right)=(-1)^{m}\left(\begin{array}{c}
m+2 k-1 \\
m
\end{array}\right)
$$


another representation of (3.1) is valid:

$$
\mathcal{G}(\lambda, n, k)=\sum_{m=0}^{\infty}(-1)^{m}\left(\begin{array}{c}
m+2 k-1 \\
m
\end{array}\right) L_{m+k}(n) \lambda^{m+k} .
$$

Combining (1.1) with (3.1) we get

$$
\mathcal{G}(\lambda, n, k)=\frac{1}{n} \sum_{d \mid n} \mu\left(\frac{n}{d}\right) \sum_{m=0}^{\infty}\left(\begin{array}{c}
-2 k \\
m
\end{array}\right) \lambda^{m+k}(m+k)^{d} .
$$

Using (2.4) in the above equation yields

$$
\mathcal{G}(\lambda, n, k)=\frac{1}{n} \sum_{d \mid n} \mu\left(\frac{n}{d}\right) \zeta_{w}(-d, k ; \lambda) .
$$

Finally, combining (2.6) with the above equation we obtain the following result:

THEOREM 3.2. Let $n$ be a positive integer. Then we have

$$
\mathcal{G}(\lambda, n, k)=\frac{1}{n} \sum_{d \mid n} \mu\left(\frac{n}{d}\right) W_{d}^{(k)}(\lambda) .
$$

By substituting $k=1$ into (3.2), we get the corollary:

COROLlary 3.3.

or

$$
\mathcal{G}(\lambda, n, 1)=\sum_{m=0}^{\infty}(-1)^{m}(m+1) L_{m+1}(n) \lambda^{m+1}
$$

$$
\mathcal{G}(\lambda, n, 1)=\frac{1}{n} \sum_{d \mid n} \mu\left(\frac{n}{d}\right) W_{d}(\lambda) .
$$

If $n$ is replaced by a prime number $p$, then (3.2) reduces to the following:

COROLLARY 3.4. Let $p$ be a prime number. Then we get

$$
\mathcal{G}(\lambda, p, k)=\frac{W_{p}^{(k)}(\lambda)-W_{1}^{(k)}(\lambda)}{p} .
$$

REMARK 3.5. Let $k=1$ in (3.3). Then, for $p=2$ and $p=3$ it reduces to

and

$$
\mathcal{G}(\lambda, 2,1)=\frac{\lambda^{2}(\lambda-2)}{(\lambda+1)^{4}}
$$

$$
\mathcal{G}(\lambda, 3,1)=\frac{4 \lambda^{2}(\lambda-1)}{(\lambda+1)^{5}},
$$

respectively.

Now, we define of a new family of polynomials $\mathcal{L}_{n}(x, m, k)$ of degree $n$ :

DEFINITION 3.6. The polynomials $\mathcal{L}_{n}(x, m, k)$ of degree $n$ are defined by

$$
\mathcal{L}_{n}(x, m, k)=\frac{1}{n} \sum_{d \mid n} \mu\left(\frac{n}{d}\right) \sum_{j=0}^{d}\left(\begin{array}{l}
d \\
j
\end{array}\right)(m+k)^{d-j} x^{j} .
$$


If $n=p$ ( $p$ is a prime number), then (3.4) yields:

COROLLARY 3.7. Let $p$ be a prime number. Then

$$
\mathcal{L}_{p}(x, m, k)=\frac{(x+m+k)^{p}-(x+m+k)}{p} .
$$

We also define a power series involving the polynomials $\mathcal{L}_{n}(x, m, k)$ :

Definition 3.8. Let $\lambda \in \mathbb{C}$ with $|\lambda|<1$, and let $n$ be a positive integer. We define

$$
\mathcal{H}(x ; \lambda, n, k)=\sum_{m=0}^{\infty}\left(\begin{array}{c}
-2 k \\
m
\end{array}\right) \mathcal{L}_{n}(x, m, k) \lambda^{m+k} .
$$

Combining (3.5) with (3.4) yields

$$
\mathcal{H}(x ; \lambda, n, k)=\frac{1}{n} \sum_{d \mid n} \mu\left(\frac{n}{d}\right) \sum_{m=0}^{\infty}\left(\begin{array}{c}
-2 k \\
m
\end{array}\right) \lambda^{m+k}(x+m+k)^{d} .
$$

By using (2.5) in the above equality, we obtain

$$
\mathcal{H}(x ; \lambda, n, k)=\frac{1}{n} \sum_{d \mid n} \mu\left(\frac{n}{d}\right) \zeta_{w}(-d, x, k ; \lambda) .
$$

Finally, with (2.7) the last equality yields the following result:

THEOREM 3.9. We have

$$
\mathcal{H}(x ; \lambda, n, k)=\frac{1}{n} \sum_{d \mid n} \mu\left(\frac{n}{d}\right) W_{d}^{(k)}(x ; \lambda) .
$$

REMARK 3.10. Substituting $k=1$ into (3.6) we have

$$
\mathcal{H}(x ; \lambda, n, 1)=\frac{1}{n} \sum_{d \mid n} \mu\left(\frac{n}{d}\right) W_{d}(x ; \lambda) .
$$

In addition, for $x=0$ it reduces to

$$
\mathcal{H}(0 ; \lambda, n, k)=\mathcal{G}(\lambda, n, k) .
$$

By combining (3.5) and (3.6), we obtain a connection between the polynomials $\mathcal{L}_{n}(x, m, k)$ and $W_{d}^{(k)}(x ; \lambda)$ :

COROLlary 3.11. We have

$$
\sum_{m=0}^{\infty}\left(\begin{array}{c}
-2 k \\
m
\end{array}\right) \mathcal{L}_{n}(x, m, k) \lambda^{m+k}=\frac{1}{n} \sum_{d \mid n} \mu\left(\frac{n}{d}\right) W_{d}^{(k)}(x ; \lambda) .
$$

4. A modification of the recurrence formula for the numbers $W_{n}^{(k)}(\lambda)$ and algorithms. In order to find the numerical values of the functions $\mathcal{G}(\lambda, n, k)$, in this section we provide some computational algorithms for the numbers $W_{n}(\lambda)$ and $W_{n}^{(k)}(\lambda)$.

For $n \geq 1$, a modification of (2.3) is given by

$$
W_{n}(\lambda)=W_{0}(\lambda) \sum_{m=0}^{n-1}\left[(-1)^{n-m+1} \lambda^{-1}-\lambda\right]\left(\begin{array}{c}
n \\
m
\end{array}\right) W_{m}(\lambda) .
$$


By using (4.1), we state Algorithm 1 for computing the numbers $W_{n}(\lambda)$.

$\overline{\text { Algorithm } 1 \text { Let } n \text { be a nonnegative integer and } \lambda \in \mathbb{C} \text {. This algorithm will return the numbers }}$ $W_{n}(\lambda)$ recursively.

procedure $\mathbf{W} \_$APOSTOL_TYPE_NUM $(n$ : nonnegative integer, $\lambda)$

Begin

Local variable $m$ : positive integer

if $n=0$ then

else

return $\lambda /$ power $(\lambda+1,2)$

return $\mathrm{W} \_\mathrm{APOSTOL}$ TYPE_NUM $(0, \lambda)$

$\hookrightarrow * \operatorname{sum}\left(((1 / \lambda) * \operatorname{power}(-1, n-m+1)-\lambda) * \operatorname{Binomial} \_\operatorname{Coef}(n, m)\right.$

end if

$\hookrightarrow *$ W_APOSTOL_TYPE_NUM $(n-m, \lambda), m, 1, n)$

end procedure

We provide some numerical values of the numbers $W_{n}(\lambda)$ computed by Algorithm 1:

$$
\begin{array}{lll}
W_{1}\left(\frac{1}{4}\right)=0.096, & W_{2}\left(\frac{1}{4}\right)=0.0064, & W_{3}\left(\frac{1}{4}\right)=-0.08832 \\
W_{4}\left(\frac{1}{4}\right)=-0.11648, & W_{5}\left(\frac{1}{4}\right)=0.059136, & W_{6}\left(\frac{1}{4}\right)=0.5126656 .
\end{array}
$$

By setting $c=k-1$ and $d=1$ in (2.2), we also have the following recurrence relation for the numbers $W_{n}^{(k)}(\lambda)$ :

$$
W_{n}^{(k)}(\lambda)=\sum_{m=0}^{n}\left(\begin{array}{c}
n \\
m
\end{array}\right) W_{m}^{(k-1)}(\lambda) W_{n-m}(\lambda) .
$$

By using (4.2), we obtain Algorithm 2 for computing the numbers $W_{n}^{(k)}(\lambda)$.

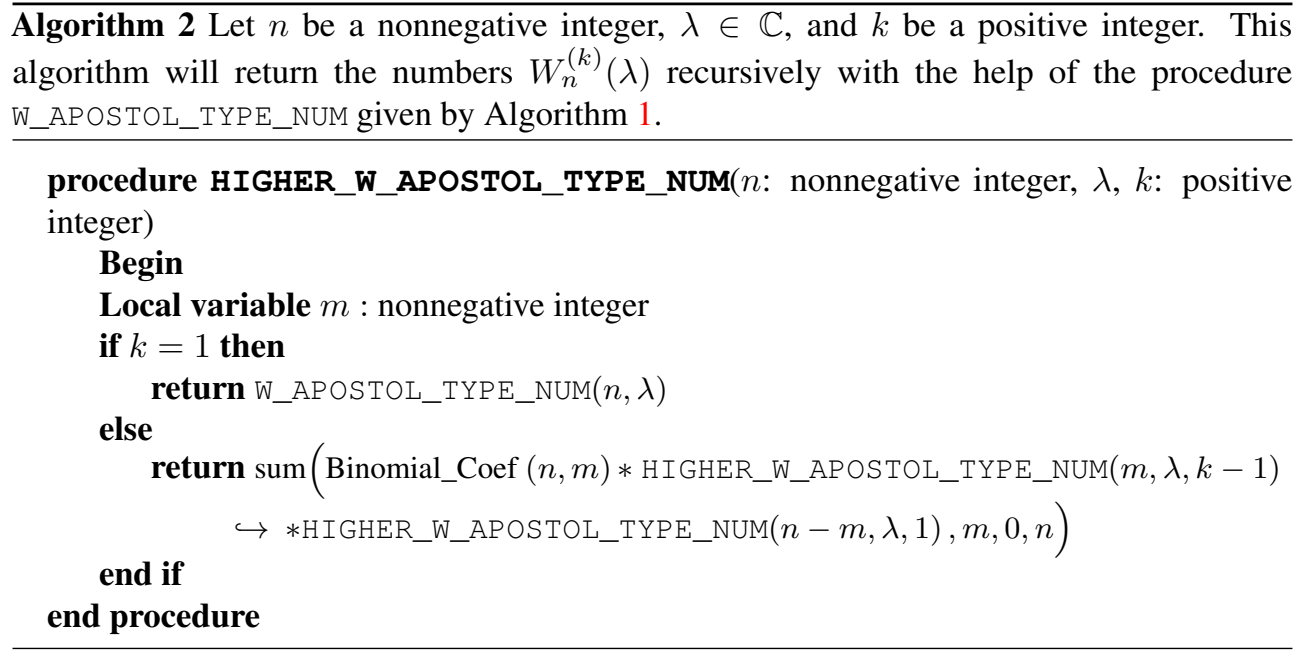




\section{ETNA}

Kent State University and

Johann Radon Institute (RICAM)

Some numerical values of the numbers $W_{n}^{(k)}(\lambda)$ computed by Algorithm 2 are as follows:

$$
\begin{aligned}
& W_{0}^{(2)}\left(\frac{1}{2}\right)=0.0493827160494 \\
& W_{0}^{(3)}\left(\frac{1}{3}\right)=0.006591796875 \\
& W_{1}^{(2)}\left(\frac{1}{2}\right)=0.0329218106996
\end{aligned}
$$

By using (3.2) related to the numbers $W_{n}^{(k)}(\lambda)$ and the Möbius function $\mu(n)$, we can also state Algorithm 3 for computing the values of the functions $\mathcal{G}(\lambda, n, k)$.

Algorithm 3 Let $\lambda \in \mathbb{C}$ with $|\lambda|<1, n$ be a nonnegative integer, and $k$ be a positive integer. This algorithm will return $\mathcal{G}(\lambda, n, k)$ given by (3.2) with the help of the procedure HIGHER_W_APOSTOL_TYPE_NUM given by Algorithm 2 and the Möbius function denoted as the procedure Mobius_Func.

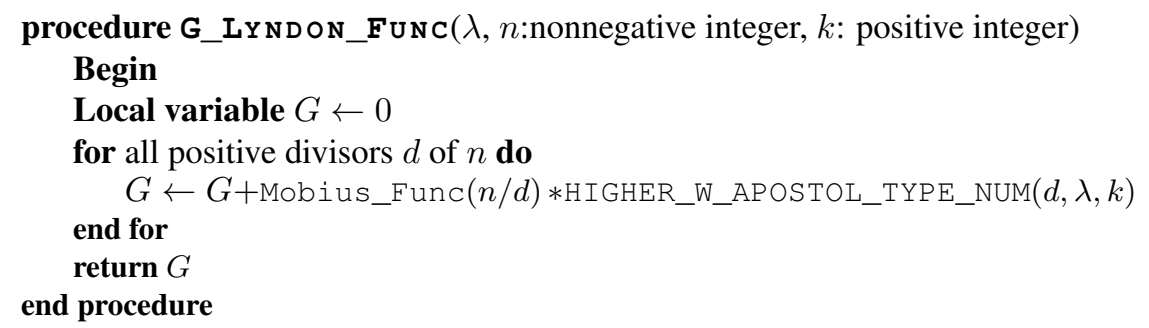

A computation by Algorithm 3 yields some values for the functions $\mathcal{G}(\lambda, n, k)$ :

$$
\begin{array}{llrl}
\mathcal{G}\left(\frac{1}{2}, 2,1\right) & =-0.0740740740741, & \mathcal{G}\left(-\frac{1}{2}, 2,1\right)=-10 \\
\mathcal{G}\left(\frac{1}{2}, 3,1\right)=-0.0658436213992, & \mathcal{G}\left(-\frac{1}{2}, 3,1\right)=-48 \\
\mathcal{G}\left(\frac{1}{2}, 5,1\right)=0.0658436213992, & \mathcal{G}\left(-\frac{1}{2}, 5,1\right)=-1872 .
\end{array}
$$

5. Numerical evaluation of the numbers $W_{n}(\lambda)$ and $W_{n}^{(k)}(\lambda)$ and the functions $\mathcal{G}(\lambda, \boldsymbol{n}, \boldsymbol{k})$. In this section, by using our algorithms, we not only provide plots for the numbers $W_{n}(\lambda)$ and the functions $\mathcal{G}(\lambda, n, k)$, but also give some numerical evaluations related to the numbers $W_{n}(\lambda)$, the numbers $W_{n}^{(k)}(\lambda)$, and the functions $\mathcal{G}(\lambda, n, k)$. Moreover, we present some illustrations and observations on the approximation of the functions $\mathcal{G}(\lambda, p, 1)$ by rational functions that can provide ideas for a reduction of the algorithmic complexity of our algorithms.

We assume that $\lambda$ is a real number. Firstly, by using Algorithm 1, we plot the numbers $W_{n}(\lambda)$ in the cases of $n=0,1, \ldots, 6$ and for varying $\lambda \in\left[\frac{1}{100}, \frac{1}{10}\right]$. It is clear that the $W_{n}(\lambda)$ are rational functions of the variable $\lambda$. Thus, the curves in Figure 5.1 provide some information for analysing some characteristics of the rational functions $W_{n}(\lambda)$. 


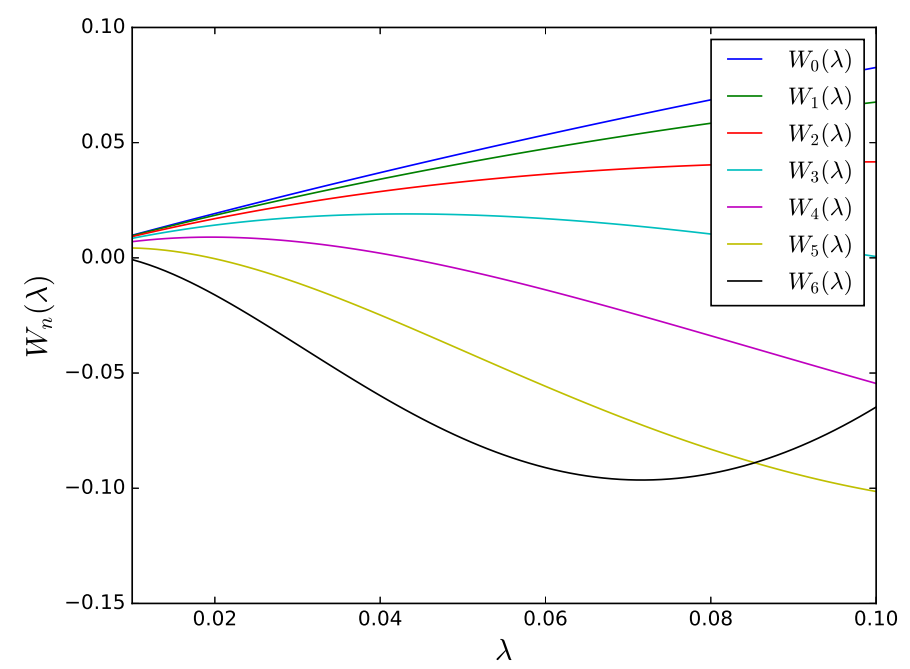

FIG. 5.1. Graphs of the numbers $W_{n}(\lambda)$ for $n=0,1, \ldots, 6$ and varying $\lambda \in\left[\frac{1}{100}, \frac{1}{10}\right]$.

Graphs of the functions $\mathcal{G}(\lambda, p, 1)$ for the prime numbers $p=2,3,5,7$ and $\lambda \in[0,5]$ obtained by Algorithm 3 are presented in Figure 5.2. This figure demonstrates the effects of the prime numbers on the shape of the curve for the selected range $\lambda \in[0,5]$, and these curves provide information for analysing some characteristics of the functions $\mathcal{G}(\lambda, p, 1)$.

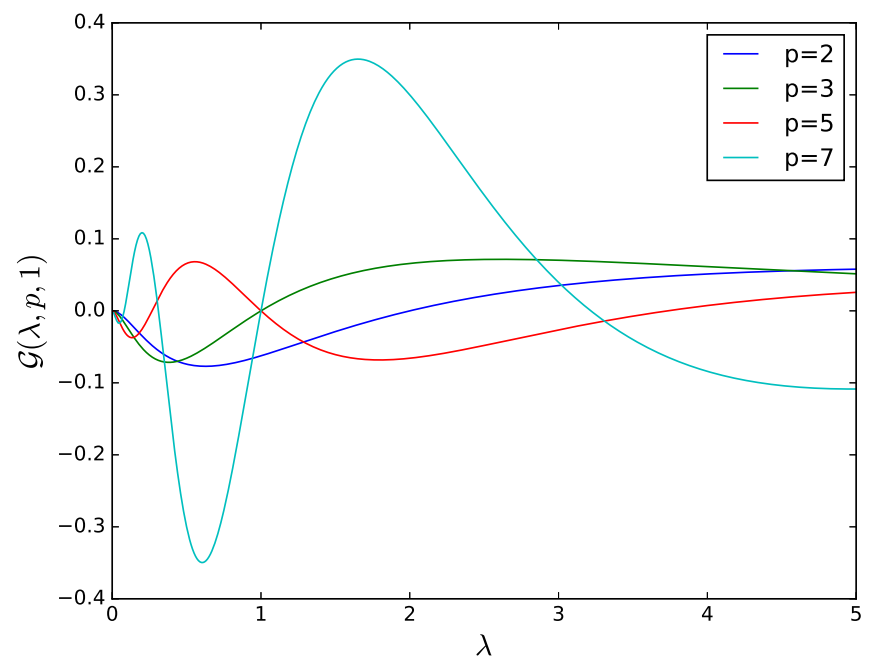

FIG. 5.2. Graphs of the functions $\mathcal{G}(\lambda, p, 1)$ for varying prime numbers and $\lambda \in[0,5]$.

Now, we provide an approximation to the functions $\mathcal{G}(\lambda, p, 1)$ by rational functions with small errors to give ideas for a reduction of the algorithmic complexity of Algorithm 3 for prime numbers. 
Let us assume that $|\lambda|<1$, and let $p$ be a prime number. It is clear that the numbers $W_{n}(\lambda)$ are rational functions of the variable $\lambda$. Therefore, by setting

$$
\mathcal{G}_{A}(\lambda, p)=\frac{W_{p}(\lambda)}{p},
$$

the following inequality holds true:

$$
\left|\mathcal{G}(\lambda, p, 1)-\mathcal{G}_{A}(\lambda, p)\right| \leq \varepsilon_{p}
$$

where $\varepsilon_{p}=1 / p$.

We present some plots and numerical experiments in order to illustrate the approximations of the functions $\mathcal{G}(\lambda, p, 1)$ by the rational functions $\mathcal{G}_{A}(\lambda, p)$ with an error less than $\varepsilon_{p}=1 / p$. The rational functions $\mathcal{G}_{A}(\lambda, p)$ for four different prime numbers $(p=3,5,7,11)$ are represented by red lines in Figure 5.3. According to (5.1) for sufficiently large prime numbers $p, \varepsilon_{p}$ tends to zero, and the curves of the functions $\mathcal{G}(\lambda, p, 1)$ and $\mathcal{G}_{A}(\lambda, p)$ tend to overlap. This indicates that by using the rational functions $\mathcal{G}_{A}(\lambda, p)$ instead of $\mathcal{G}(\lambda, p, 1)$, Algorithm 3 can operate more efficiently for sufficiently large prime numbers.
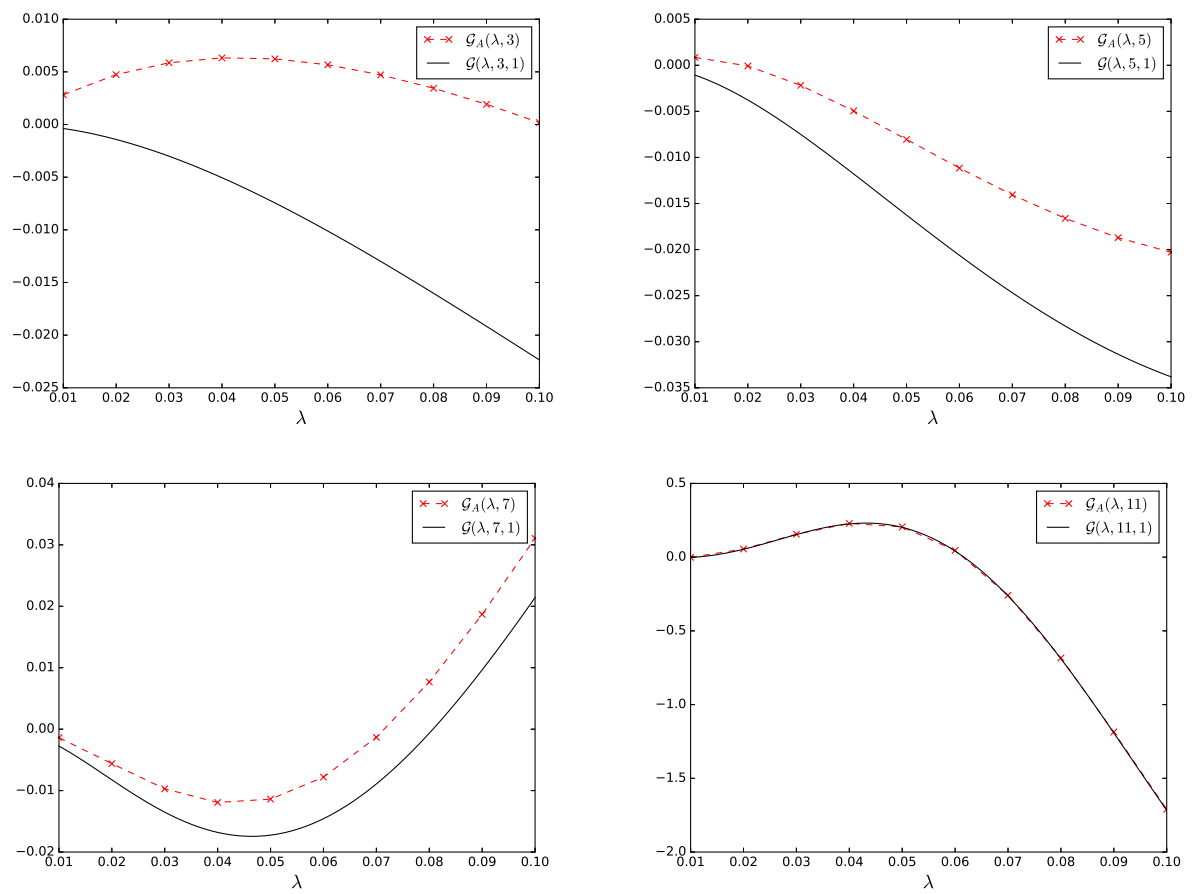

FIG. 5.3. Approximations of the function $\mathcal{G}(\lambda, p, 1)$ by the rational function $\mathcal{G}_{A}(\lambda, p)$ in the cases: $(a) p=3$, $\varepsilon_{3}=1 / 3$; (b) $p=5, \varepsilon_{5}=1 / 5$; (c) $p=7, \varepsilon_{7}=1 / 7$; (d) $p=11, \varepsilon_{11}=1 / 11$.

REMARK 5.1. In [10], Kucukoglu et al. constructed generating functions for the $k$-ary Lyndon words having prime number length with the help of the Apostol-Bernoulli numbers and other special numbers. Moreover, they gave an approximation to these generating functions by rational functions of the Apostol-Bernoulli numbers. In this paper, we give approximations 


\section{ETNA}

Kent State University and

Johann Radon Institute (RICAM)

for functions representing our special power series by rational functions associated with Apostol-type numbers by using similar techniques as the ones in [10].

\section{REFERENCES}

[1] T. M. Apostol, On the Lerch zeta function, Pacific J. Math., 1 (1951), pp. 161-167.

[2] - Introduction to Analytic Number Theory, Springer, New York, 1998.

[3] J. Berstel AND D. PERrin, The origins of combinatorics on words, European J. Combin., 28 (2007), pp. 996-1022.

[4] T. W. Cusick And P. StaniCA, Cryptographic Boolean Functions and Applications, Elsevier, Amsterdam, 2009.

[5] T. KIM, S.-H. RIM, Y. SIMSEK, AND D. KIM, On the analogs of Bernoulli and Euler numbers, related identities and zeta and L-functions. J. Korean Math. Soc., 45 (2008), pp. 435-453.

[6] I. KUCUKOGLU, A. BAYAD AND Y. SIMSEK, $k$-ary Lyndon words and necklaces arising as rational arguments of Hurwitz-Lerch zeta function and Apostol-Bernoulli polynomials, Mediterr. J. Math., 14 (2017), Art. No., 223, 16 pages.

[7] I. KUCUKOGLU AND Y. SIMSEK, On interpolation functions for the number of $k$-ary Lyndon words associated with the Apostol-Euler numbers and their applications, RACSAM Rev. R. Acad. A., (2017), pp. 1-17. DOI: $10.1007 / \mathrm{s} 13398-017-0471-\mathrm{y}$

[8] - Remarks on recurrence formulas for the Apostol-type numbers and polynomials, Adv. Stud. Contemp. Math. (Kyungshang), 28(4) (2018), pp. 643-657.

[9] I. Kucukoglu, Y. SimseK, AND H. M. SRIVASTAVA, A new family of Lerch-type zeta functions interpolating a certain class of higher-order Apostol-type numbers and Apostol-type polynomials, Quaest. Math., (2018), pp. 1-14. DOI: 10.2989/16073606.2018.1459925

[10] I. Kucukoglu, G. V. Milovanović, AND Y. Simsek, Analysis of generating functions for special words and numbers with algorithms for their computation, Preprint, 2017.

[11] M. Lothaire, Combinatorics on Words, Cambridge University Press, Cambridge, 1997.

[12] Q.-M. Luo And H. M. SRIVAstava, Some generalizations of the Apostol-Bernoulli and Apostol-Euler polynomials, J. Math. Anal. Appl., 308 (2005), pp. 290-302.

[13] Q.-M. LuO, Apostol-Euler polynomials of higher order and Gaussian hypergeometric functions, Taiwanese J. Math., 10 (2006), pp. 917-925.

[14] Y. SIMSEK, Computation methods for combinatorial sums and Euler type numbers related to new families of numbers, Math. Methods Appl. Sci., 40 (2017), pp. 2347-2361.

[15] - Interpolation function for the families of numbers related to the Apostol-type numbers, in International Conference of Numerical Analysis and Applied Mathematics (ICNAAM 2017), AIP Conf. Proc., Vol. 1978, AIP Publishing, Melville, 2018, Art. Id. 040012. Doi:10.1063/1.5043696

[16] H. M. SRIVAStaVA, Some generalizations and basic (or q-)extensions of the Bernoulli, Euler and Genocchi polynomials, Appl. Math. Inf. Sci., 5 (2011), pp. 390-444.

[17] H. M. SRIVASTAVA, T. KIM, AND Y. SimSEK, q-Bernoulli numbers and polynomials associated with multiple q-zeta functions and basic L-series, Russ. J. Math. Phys., 12 (2005), pp. 241-268.

[18] H. M SRIVAstaVA AND J. CHOI, Zeta and q-Zeta Functions and Associated Series and Integrals, Elsevier Amsterdam, 2012. 